\title{
Development of Virtual Museum Model for Youth
}

\author{
Unchana Klentien
}

\begin{abstract}
The research provided guidance of the key elements on design and development of virtual museum which is a critical learning resource supporting learning experience outside the classroom for youth. The objective of this research is to develop a prototype of virtual museums for youth and evaluate the efficiency of the prototype. The research comprised three steps: 1) studying demands and expectations of virtual museums for youth by examining documents and researches about virtual museums, visiting 10 museums in Bangkok and 20 virtual museums in Thailand and worldwide, and conducting a questionnaire survey on 400 university students about their expectations of virtual museums; 2) developing a virtual museum website prototype and trialing it with three experts and 10 university students for improvement recommendation; and 3) having five experts evaluate the quality and efficiency of the improved prototype. The study showed that virtual museums for youth should consist of five key elements: 1) efficiency of website's function, 2) the website itself, 3) type of media used in the virtual museum, 4) type of tools used in the virtual museum, and 5) evaluation of the virtual museum. The virtual museum prototype received a highest level score from the experts (mean $=4.56$ ) and a high level score from the university students $($ mean $=4.43)$. Moreover, evaluation results from the other five experts showed that all elements of the improved prototype were appropriate.
\end{abstract}

Index Terms-Museum, virtual museum, youth, self-directed learning.

\section{INTRODUCTION}

Museum is a place where important information is gathered and important objects are exhibited, for the sake of education, research, and entertainment. Museums are valuable sources of self-directed learning for youth on a great variety of topics. With the advent of the 21 st century, learning has changed significantly and become more open. Learners no longer have to wait for knowledge from teachers only, but can seek knowledge themselves. And this can lead to lifelong learning [1]. Application of virtual reality technology to develop virtual local museums, which can display still pictures, motion pictures, and 360-degree motion pictures, does not only create environments that can promote learning proficiency, but also allows learners to engage in self-directed learning anywhere and anytime regardless of the limitation of space and time [2]. The Covid-19 global health crisis has altered the way of learning from classroom to online and the way of working from office working to working from home. Likewise, many museums and cultural institutions have quickly adapted to the current situation by creating virtual access to their exhibits. Many cultural institutions have seized this occasion to share more content

Manuscript received August 23, 2021; revised November 5, 2021.

Unchana Klentien is with Srinakharinwirot University, Bangkok, Thailand (e-mail: unchana@g.swu.ac.th). and reach a wider audience online or through social networks [3].

Virtual museums exhibit content, e.g. texts, pictures, motion pictures, audio records, and videos, in a digital form and audiences can visit them via the Internet from anywhere, at anytime [4]. However, virtual museums have not received much attention because people usually gain better learning experience with all five senses in physical learning environments. Maria Kampouropoulou et al. [5] studied views of primary school students on the use of a virtual education museum and found that part of the students preferred visiting physical museums. Yet, there was also a group of students who thought a virtual museum was interesting and could help them understand history better than visiting the real sites. This finding was in line with a research of Rattanaporn Chiengkham et al. [6] on the 3D virtual exhibition about the social and cultural development in Thailand at Maha Chakri Sirindhorn Anthropology Centre, which showed that the learning outcome from a 3D virtual exhibition was better than the learning outcome from a permanent exhibition. It was because, at a permanent exhibition students had to rely on the lecturer and there was also limitation of time for exploring and areas for exhibition. Moreover, visiting an exhibition with a large group of people made it difficult for students to concentrate. In contrast for a virtual exhibition, students could explore any exhibitions worldwide without those limitation and distraction.

Unfortunately, museums are only popular among those who are enthusiastic about art, and the number of people visiting museums is still few. A German philosopher, Alexander Baumgaten, argued that aesthetics was knowledge from experience and an intuition in judging the beauty of art without the interference of reasons [7]. Therefore, virtual museums can act as another channel to link aesthetics with appreciation of beauty in order to bring art into everyday life of people in the 21 st century. Kritchanat Santawee and Sammiti Sukbunjhong [8] suggested that art museums should also provide virtual museum service via the Internet. Likewise, Dendeon Lertthayakul [9] found that development of a virtual local museum could improve learning outcome of students. In addition, a research of Nitisak Charoenroop [10] on application of augmented reality to promote tourism information showed that tourists had the highest level of satisfaction about the application and the idea of using augmented reality media in the future.

Since virtual reality technology is important to the development of learning for people in the 21 st century to mitigate the limitation of time and space for learning, this research aimed to develop a model of virtual museums to fill in the learning gap regarding aesthetics and youth. This study examined key elements of existing virtual museums and developed a prototype of virtual museums to be used for future development or adaptation by private or public sectors. 
The Archive of Srinakharinwirot University was used as a model for developing the virtual museum prototype in this research.

\section{ReSEARCH APPROACH AND MethodOlOGY}

\section{A. Research Purpose}

The objective of this research is to study and develop a prototype of virtual museums for youth and evaluate the efficiency of the prototype.

\section{B. Sample Groups and Participants}

There were three sample groups in this study. The first sample group consisted of 400 university students aged between 18 and 25 from the science and social science fields of study. These samples were asked to answer a questionnaire about their expectations of virtual museums. The samples were chosen by accidental sampling from four universities, which were Srinakharinwirot University and Chulalongkorn University in Bangkok, Chiang Mai University in Chiang Mai Province, and Mahasarakham University in Maha Sarakham Province. The second sample group consisted of three experts on virtual reality design, art content, and/or museum management and ten university students. These samples were asked to trial and comment on the developed virtual museum prototype for youth. The third sample group consisted of five experts on virtual reality design, art content, and museum management. These samples were asked to evaluate the quality and efficiency of the improved virtual museum prototype for youth.

\section{Ethical Issues}

Information and identity of the students participating in this research was treated as confidential and used for the study and statistical purpose only.

\section{Research Methods}

The research methodology comprised three steps. The first step was studying demands and expectations of virtual museums for youth by 1) examining documents and researches about virtual museums, 2) visiting 10 museums in Bangkok to examine their media use, technology, and designs, 3) visiting websites of 20 virtual museums in Thailand and worldwide to examine their efficiency, media use, virtual museum tools, designs, and assessment, and 4) conducting a five-Likert scale questionnaire survey on 400 university students about their expectations of virtual museums. The data of respondents' general information and internet use behavior were analyzed by frequency count and percentages, whereas, the data on pattern of virtual museum were analyzed by mean and standard deviation. The interpretation of mean score was specified as following: $1.00-1.50=$ very low, $1.51-2.50=$ low, $2.51-3.50=$ average, 3.51-4.50 = high, and 4.51-5.00 = very high.

The second step was developing a virtual museum website prototype that included pictures, 360-degree panoramic images, and virtual reality technology by using the Archive of Srinakharinwirot University as a model. The developed prototype was subsequently trialed with three experts and ten university students for recommendation and improvement.
The final step was having five experts evaluate the improved prototype in terms of the website format, media presentation, virtual museum tools, and quality and efficiency assessment. The Index of Item-Objective Congruence: IOC was used to evaluate the virtual museum model validity based on the score range from -1 to +1 was defined as following: $+1=$ Congruent, $0=$ Questionable, and $-1=$ Incongruent. The questions which had scores lower than 0.5 were revised.

\section{FINDINGS AND ANALYSIS}

1) Findings from the analysis of virtual museums in Thailand and worldwide and the survey of expectations of virtual museums for youth from 400 university students can be concluded as follows.

a) Online media use behavior - The majority or 174 people of the sample group (43.50 percent) had access to online media and the Internet through iOS smartphones, followed by 93 people (23.25 percent) through Android smartphones, 79 people (19.75 percent) through iPad, and 54 people (13.50 percent) through other devices. In terms of online media and the Internet use, the majority or 289 people of the sample group (72.25 percent) used online media in a form of applications most frequently, while the other 111 people (27.75 percent) used online media in a form of websites most frequently. The majority of the sample group (129 people or 32.25 percent) used online media for a continuous period of 31-60 minutes per time, followed by 91 - 120 minutes per time (106 people or 26.50 percent).

The majority of the sample group had never visited or used virtual museums via online media and the Internet (217 people or 54.25 percent). Only 27.50 percent (110 people) of the sample group used to visit or get information from virtual museums, while 18.25 percent ( 73 people) were not sure or did not remember if they used to visit virtual museums or not. Yet, the majority of the sample group were interested in using online virtual museums (314 people or 78.50 percent) and thought online virtual museums could provide knowledge like traditional physical museums (319 people or 79.75 percent).

b) Expectations of virtual museums:

Learning management in virtual museums - The sample group answered the questions with a high level score the most (mean $=4.46)$. The item that received the highest score was "Virtual museums should employ diversified media technologies to portray knowledge". The item that received the second highest score was "Virtual museums should provide entertainment and knowledge at the same time".

Efficiency of the virtual museums - The sample group answered the questions with a highest level score the most (mean $=4.60)$. The item that received the highest score was "Virtual museums should be accessible from a variety of devices such as a personal computer, tablet, or mobile phone". The item that received the second highest score was "Virtual museums should be quick to load and display for prompt use".

Types of media and tools used in virtual museums - The 
sample group answered the questions with a high level score the most $($ mean $=4.40)$. The item that received the highest score was "Audience should be able to visit virtual museums freely". The item that received the second highest score was "Virtual museums should support more than one languages, with language options provided for audience to choose".

In addition, the website analysis showed that important tools for virtual museums included registration tools, search engine, social media tools such as Facebook, Twitter, and YouTube, and online discussion tools. Moreover, most virtual museums offered 360-degree panoramic images for 360-degree exhibition tours. Texts, pictures, or videos were used in the description and display of exhibitions. Meanwhile, photographic slides were used as thumbnail images to link to different exhibition rooms. Virtual reality (VR) techniques with and without headset goggles were used as well as $3 \mathrm{D}$ and 360-degree image techniques to display models, buildings, sculptures, skeletons, or antiquities. Visitors could explore virtual exhibitions freely by using the zoom-in button and the map and navigator tools provided in the websites. There were also links to the museum's websites for further studies.

Only some of the websites offered audio descriptions with a speaker button. Audience could choose to turn on or turn off the sound with the button. There were also a help button, directional arrows, share button, download button, and screenshot button.

Designs of the virtual museums - The sample group answered the questions with a high level score the most (mean $=3.77)$. The item that received the highest score was "Fonts used in virtual museums should be easy to read and clear". The item that received the second highest score was "Virtual museums should use dark text on light background such as dark blue text on a white background".

The website analysis showed that exhibition rooms in virtual museums used warm color tone, cool color tone, and neutral color tone depending on the content in each section. The websites used maximum two font styles and maximum three display colors, including the color of the background. Text in each text box was left aligned or centered. Text boxes were placed on the left or right of images or objects, depending on the positions of those objects.

Evaluation of the virtual museums - The sample group answered the questions with a high level score the most (mean $=4.28)$. The website analysis showed that most virtual museums did not have an evaluation section for users to comment or provide feedback for improvement. However, some virtual museums had a Frequently-Asked-Question (FAQ) section or online discussion section instead.

2) Findings from the trial of the developed virtual museum prototype for youth showed that the three experts gave a highest level score for the prototype in overall (mean = 4.56) and the ten university students gave a high level score for the prototype in overall (mean = 4.43). Both groups agreed that in the current situation of Covid-19, museums should use media and technology to develop virtual museums that could provide similar user experience as physical museums to visitors.

3) Findings from the evaluation of quality and efficiency of the virtual museum prototype for youth showed that the five experts viewed that the improved prototype were appropriate in overall $(\mathrm{IOC}=1)$. Only the elements of $3 \mathrm{D}$ object images and maps received 0.6 of IOC and needed further improvement. The experts suggested that the $3 \mathrm{D}$ object images should have realistic texture and colors, so that they were more interesting and attractive for youth. Regarding this, the researcher still used existing white 3D object images from the Archive of Srinakharinwirot University but added realistic images of the building under the 3D image model section on the website instead. The experts also suggested that there should be a pointer or pin on the map that indicated a current location of users when exploring the virtual museums. The researcher added the location indicator pin on the map accordingly. After finishing the three steps of the research, five key elements of virtual museums for youth were concluded as shown in Fig. 6. In addition, Fig. 1-5 show the pictures of The Archive of Srinakharinwirot University, which was recreated in a virtual model by using those five elements.

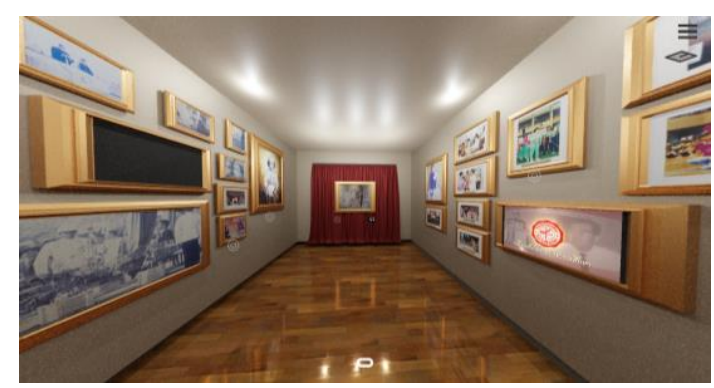

Fig. 1. Hall of Fame at the Archive in a 360-degree panoramic image.

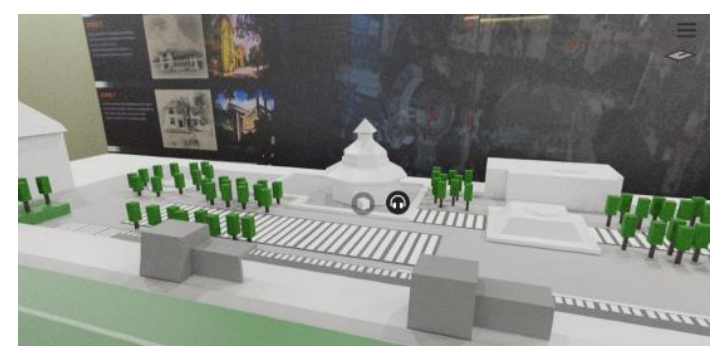

Fig. 2. 3D version of the university's model.

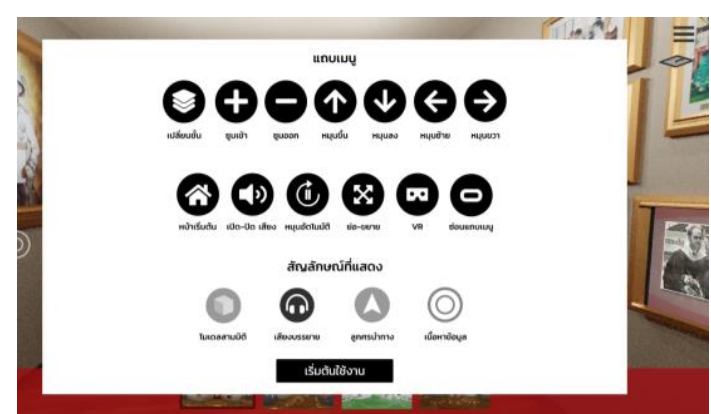

Fig. 3. The virtual museum's guiding manual.

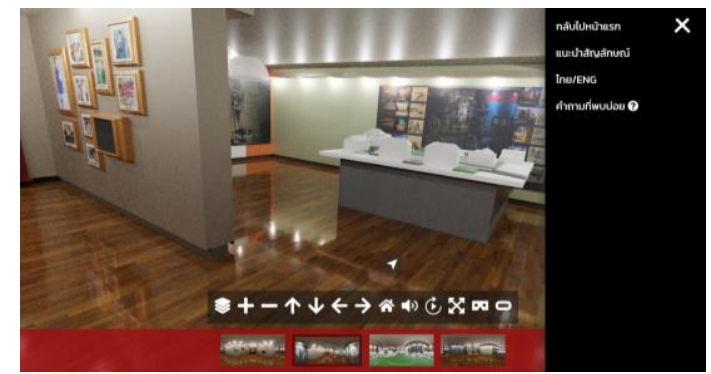

Fig. 4. The navigator of the virtual model. 


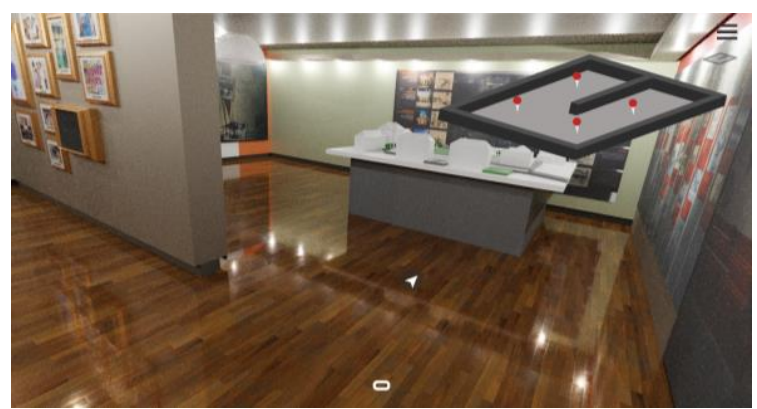

Fig. 5. Map and directional arrow.

5 Elements of Virtual Museum Model for Youth

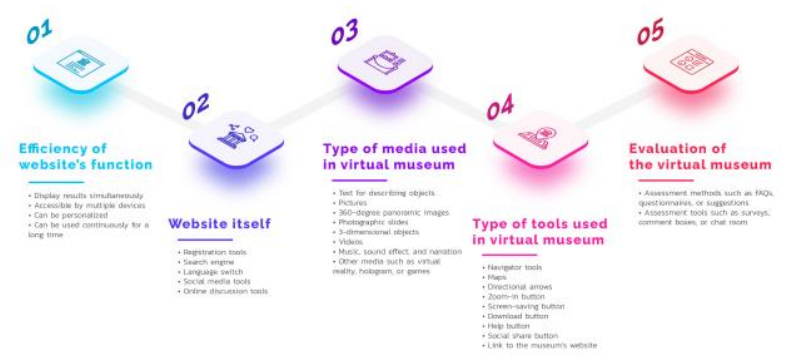

Fig. 6. Five elements of virtual museums for youth.

\section{DISCUSSIONS AND CONCLUSIONS}

Virtual museums allow youth to get access to learning sources from anywhere at anytime, especially during the current Covid-19 situation. In Thailand, however, virtual museums are not much supported or promoted and the accessibility is relatively low. Even physical museums are only popular among certain groups of people, while others may only visit museums during school educational tours. Many museums have been forgotten. Yet, museums are a great source of knowledge for youth. They are places where evidences of human culture and evolution are stored. As museums are an innovation that can enhance learning process a user-centered approach should be adopted in museum management, by offering a variety of activities and services for a wide range of audience such as travelling exhibitions or online exhibitions [8], [11]. In addition, with the evolving communication technology, relationship building between museums and audience to improve learning experience and encouraging audience to have critical thinking and to develop knowledge by themselves are also important points to consider [12]. If virtual museums are promoted and media and technology are adopted to provide realistic experience for virtual museum users, especially youth, those users would be able to learn and understand messages from virtual museums and enjoy visiting museums at the same time [13].

Accordingly, the most important thing is to engage users with the media and allow them to explore the museums freely on their own. Dina Ismaeel and Ahlam Al-Abdullatif [14] studied the impact of an interactive virtual museum on students' attitudes toward cultural heritage education in the region of Al Hassa, Saudi Arabia, and found that 118 students who visited the virtual museum had a highest level of satisfaction. The virtual museum helped those students gain knowledge about their cultural heritage. Moreover, the interactive virtual museum fit their style of learning and interest and also provided them entertainment while learning.
This finding suggested that learners in the 21 st century are digital citizens who prefer interactive learning with the help of technology. Likewise, Ployphan Chittarach and Natirath Weeranakin [15] studied the development of a dinosaur virtual museum application with image-based environment and found that related parties would like virtual museums to provide realistic experience to users with 360-degree panoramic images and to be easily accessible from smartphones. A mobile-friendly virtual museum may suit the modern lifestyle of people these days in terms of convenience and easy access. Therefore, virtual museums should be designed to fit a variety of devices. Moreover, multimedia such as 3D images, 3D motion images, audio description, text description, and short video should be employed to enhance user experience. With virtual museums, not only do these users no longer have to travel to museums, but they can also have a close up look at the exhibits just by zooming in. As a result, there have been attempts to develop and improve virtual reality systems and panoramic image technologies on websites for the sake of education [16]. A finding from a Colorado State University's research showed that 70 percent of virtual museum visitors usually visited the real museum after [17]. Consequently, virtual museums should ensure attractive environment, content, and exhibit management, provide visual and audio interactive experience for visitors, and encourage new ideas and inspiration with augmented reality (AR) and virtual reality (VR) technology, so that visitors can enjoy full experience of the museums without error or mistake [18].

\section{RECOMMENDATIONS FOR RELATED PARTIES}

This virtual museum prototype for youth has been developed by taking into account the expectations and suggestions of university students, the target group of the museums. Related parties from the public or private sectors can apply this prototype to develop websites and virtual museums to add value and improve access of digital citizens to museums. Virtual museums can be an important source of knowledge and learning without the limitation of space and time, especially when access to physical museums is difficult because of the current Covid-19 pandemic crisis.

\section{CONFLICT OF INTEREST}

The author declares no conflict of interest.

\section{AUTHOR CONTRIBUTIONS}

The author conducted the research, analyzed the data, wrote the paper, and had approved the final version.

\section{REFERENCES}

[1] T. Uaaree. (2015). Smart classroom and learning management in the 21st century. [Online]. Available: http://uaaree.dusit.ac.th/wp-content/uploads/2015/12/article-smart-cla ssroom.pdf

[2] K. Tissana, Science of Teaching: Knowledge for Effective Learning Process Management, Bangkok: Chulalongkorn University Press, 2015.

[3] UNESCO. (May 2000). Virtual Museums on Underwater Cultural Heritage Respond to the Covid-19 Crisis. [Online]. Available: 
https://en.unesco.org/news/virtual-museums-underwater-cultural-herit age-respond-covid-19-crisis

[4] B. Bunchoo, "Model of eastern of arts and culture virtual museum," Burapha University Research Report, Chonburi: Burapha University, 2013.

[5] K. Maria et al., "Students' views on the use of a virtual educational museum," European Studies, vol. 7, no. 11, pp. 1-6, 2015.

[6] C. Rattanaporn, N. Prachyanun, and W. Panita, "The 3D virtual exhibition about the social and cultural development in Thailand Maha Chakri Sirindhorn Anthropology Centre," Technical Education Journal King Mongkut's University of Technology North Bangkok, vol. 5, no. 2, pp. 85-93, July-December 2014.

[7] H. Kai, The German Aesthetic Tradition, Printed in the United Kingdom at the University Press, Cambridge, 2001.

[8] S. Kritchanat and S. Sammiti, "Trend and the factors that affecting on aesthetics experience of viewer at contemporary art and cultural museum," Office of Contemporary Art and Culture Research Report, Bangkok: Ministry of Culture, 2017.

[9] L. Dendeon, "Development of a virtual local museum: The case study of learning center for traditional local fabrics hand-woven by Lao Ethnicities of Chee and Khrang in Suphanburi province," Nakhon Phanom University Journal, vol. 8, no. 3, pp. 52-62, September-December 2018.

[10] C. Nitisak, "Applications of augmented reality to present tourist information: A case study of Phrakaew temple, Chiangrai province, Thailand," Journal of Modern Management Science, vol. 10, no. 1, pp. 13-30, January-June 2017.

[11] P. Chaleo and S. Kullaya, "Museums: Resources center for youth socialization," Journal of Humanities and Social Sciences Thonburi University, vol. 5, no. 10, pp. 1-7, July-December 2013.

[12] K. Juthamas, "The study of learning process in museum to support lifelong learning," HRDO Journal, vol. 8, no. 1, pp. 32-59, January-June 2016

[13] K. Komsit, "A study of Thai youth motivation towards museum tourism in Bangkok metropolis and its perimeters," MSc. Thesis, Dept Sports Science., Chulalongkorn Univ., Bangkok, Thailand, 2009.

[14] I. Dina and A. Ahlam, "The impact of an interactive virtual museum on students' attitudes toward cultural heritage education in the region of
Al Hassa, Saudi Arabia," International Journal of Emerging Technologies in Learning (iJET), vol. 11, no. 4, pp. 32-39, 2016.

[15] C. Ployphan and W. Natirath, "Developing dinosaur virtual museum application with image-based environment," presented at the 14th Mahasarakham University Research Conference, Mahasarakham University, September 6-7, 2018

[16] J. Pijitra, "The dissemination of knowledge for mural painting on Rattanakosin Island by virtual museum system," Veridian e-Journal, vol. 10, no. 1, pp. 1028-1041, January-April 2017.

[17] L. Ross, E. Steven, and W. Maecella. (January 2003). Website availability and visitor motivation: An evaluation study for the Colorado Digitization Project. Fort Collins, CO: Colorado State University. [Online]. Available: https://www.researchgate.net/publication/237296455

[18] D. Marija and B. Antonija, "Virtual technology in museums and art galleries business practice - The empirical research," presented at the 7th International OFEL Conference on Governance, Management and Entrepreneurship: Embracing Diversity in Organisations, April 5-6, 2019.

Copyright (C) 2022 by the authors. This is an open access article distributed under the Creative Commons Attribution License which permits unrestricted use, distribution, and reproduction in any medium, provided the original work is properly cited (CC BY 4.0).

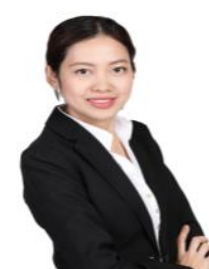

Unchana Klentien received M.Ed. in audio-visual communications from Chulalongkorn University and Ph.D. in computer education from King Mongkut's University of Technology North Bangkok, Thailand. Currently she is a lecturer in computer for communication at the College of Socia Communication Innovation, Srinakharinwiro University, and she is also working as the deputy director for the Center for Academic Services, Srinakharinwirot University. She teaches courses in technology, user interface design and website development. Her research interests are educational technology, curriculum development for both online and offline, and learning organization. 